\title{
Perspective
}

PERSPECTIVE Actualité en histoire de l'art

1 | 2021

Portugal et espaces lusophones

\section{L'azulejo portugais : patrimoine, mémoire et identité}

Une discussion entre Rosário Salema de Carvalho et Alexandre Pais, menée par Alexandra Curvelo

Rosário Salema de Carvalho, Alexandra Curvelo et Alexandre Pais

Traducteur : Élodie Dupau

\section{(2) OpenEdition}

Journals

Édition électronique

URL : https://journals.openedition.org/perspective/22400

DOI : $10.4000 /$ perspective. 22400

ISSN : 2269-7721

Éditeur

Institut national d'histoire de l'art

Édition imprimée

Date de publication : 14 octobre 2021

Pagination : 41-60

ISBN : 978-2-917902-91-2

ISSN : $1777-7852$

Référence électronique

Rosário Salema de Carvalho, Alexandra Curvelo et Alexandre Pais, «L'azulejo portugais : patrimoine, mémoire et identité », Perspective [En ligne], 1 | 2021, mis en ligne le 31 décembre 2021, consulté le 12 mai 2022. URL : http://journals.openedition.org/perspective/22400 ; DOI : https://doi.org/10.4000/ perspective. 22400 


\title{
L'azulejo portugais : patrimoine, mémoire et identité
}

\author{
Une discussion entre Rosário Salema de Carvalho \\ et Alexandre Pais, menée par Alexandra Curvelo
}

L'azulejo est l'une des expressions artistiques les plus spécifiques et singulières du Portugal. C'est d'ailleurs l'un des mots qui vient tout de suite à l'esprit lorsque l'on pense à l'art et à la culture de ce pays, ainsi qu'aux termes qui la désignent et qui la représentent. Le seul qui lui fait concurrence est, peut-être, le mot fado. Ce tandem est intéressant car les deux termes se présentent comme les éléments essentiels d'une image et d'un discours bâtis sur l'identification de traits caractéristiques de la culture portugaise, et même d'une façon $d^{\prime}$ ' " être " portugais. Porteurs d'une charge imagière et symbolique considérable, ils s'ancrent dans l'idée et l'imaginaire d'un patrimoine, d'une mémoire $^{1}$ et d'une identité culturelle nationaux ${ }^{2}$, qu'ils nourrissent à leur tour. Étant difficiles à traduire (dans le cas de fado, d'ailleurs, c'est impossible), on préfère les employer en portugais : traduire azulejo par " carreau " en français ou tile en anglais entraîne une perte et revient à éliminer dès l'énoncé la racine même de la mémoire de l'azulejo au Portugal'3.

Issu du mot arabe al-zuleij, qui signifie " pierre polie ", l'azulejo renvoie à une période où quasiment toute la péninsule Ibérique était sous domination musulmane et portait le nom d'Al-Andalus - une période qui a débuté en 711 et duré près de huit cent ans, jusqu'à la prise de Grenade, en 1492. Au Portugal, la reconquête chrétienne s'est achevée plus tôt qu'en Espagne avec la reconquête définitive de Silves en 1253, soit plus de quarante ans avant le traité d'Alcañices (1297), qui fixa les frontières entre les royaumes portugais et castillan. Les traces de la présence arabe dans la péninsule Ibérique ont cependant largement dépassé la chronologie et les frontières politiques établies par ces évènements. Profondément enracinées dans la culture et la vie péninsulaires, surtout dans sa région la plus méridionale, aujourd'hui elles ont donné lieu à une identité propre qui se manifeste dans la langue, dans la musique, dans l'architecture (fig. 1) et le travail de menuiserie qui lui est associé - les plafonds à caissons sculptés en sont un bel exemple -, dans le mobilier, dans le lien existant entre les espaces d'habitation et les cours et jardins ${ }^{4}$, et tout particulièrement dans l'azulejo et son utilisation qui n’a jamais passé de mode. 


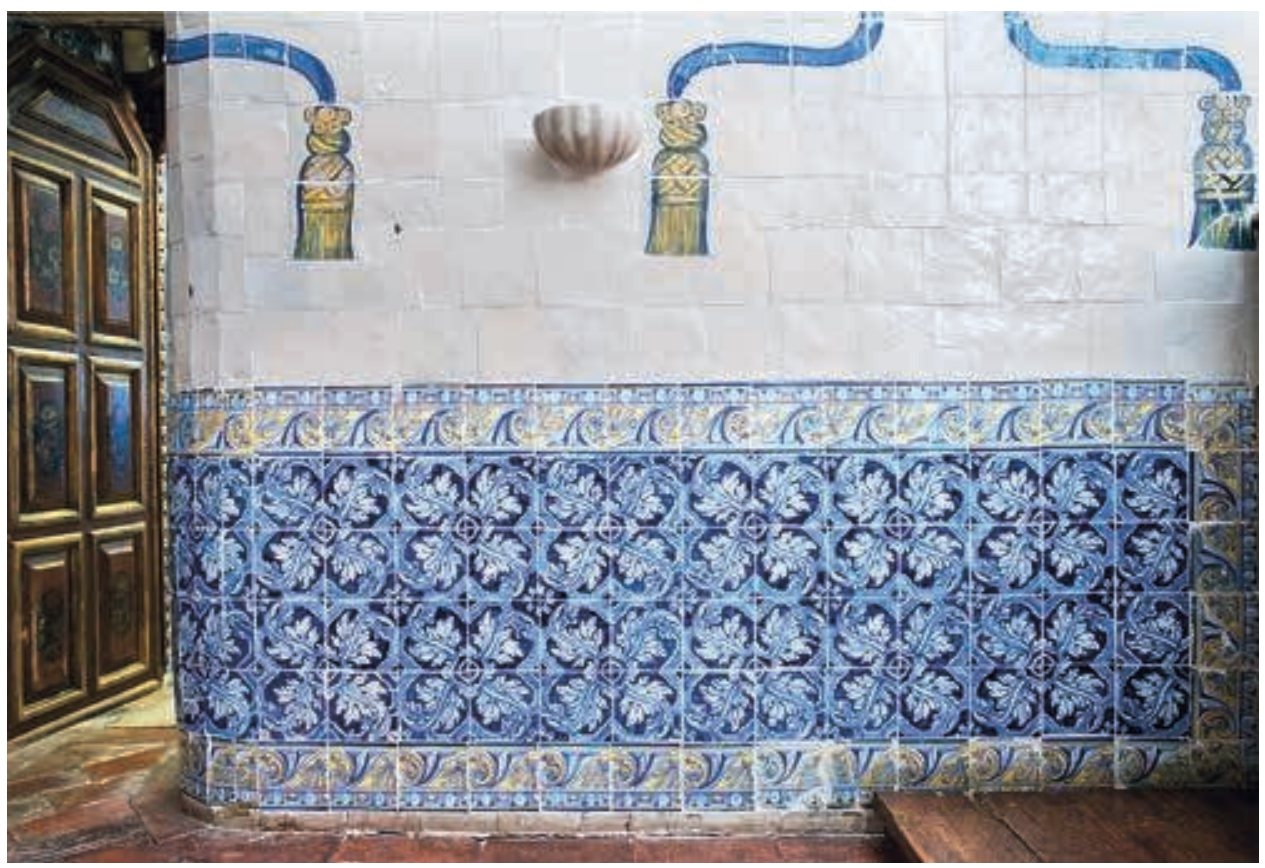

1. Plafond à caissons sculptés de la cathédrale Notre-Dame-de-l'Assomption, début du XVIe siècle, Funchal, île de Madère.

2. Décor (détail) de la chapelle du palais de Santos, actuelle ambassade de France à Lisbonne.
Ce substrat arabe de la culture péninsulaire, unique, a d'ailleurs marqué les voyageurs étrangers qui parcouraient cette vaste région aux $\mathrm{XV}^{\mathrm{e}}$ et $\mathrm{XVI}^{\mathrm{e}}$ siècles, comme Léon de Rozmital (1465-1467), Hieronymus Münzer (1494-1495) ou Claude de Bronseval $(1533)^{5}$. De fait, le phénomène mudéjar est apparu dans ce cadre politique et culturel de la péninsule Ibérique où se mêlaient les éléments chrétiens et musulmans. Si le terme, qui dérive du mot arabe mudağğan et signifie " domestiqué ", renvoie à l'origine aux musulmans devenus sujets des royaumes chrétiens, il désigne aussi un art singulier qui se manifeste différemment selon les régions ${ }^{6}$.

À partir de cette époque, l'azulejo représente l'expression matérielle de ce croisement des cultures péninsulaires. Au Portugal, son utilisation devient notable et se déploie : il voyage du continent jusqu'aux îles atlantiques (les Açores et Madère) et aux territoires d'outre-mer, en particulier au Brésil ${ }^{7}$. En parallèle, et dans une histoire à laquelle ne sont étrangers ni les développements techniques, comme l'apparition de la majolique et de la faïence au XVI ${ }^{\mathrm{e}}$ siècle, ni les mutations d'un monde de plus en plus interrelié à partir de l'époque moderne, il porte, dans son double rôle d'élément de revêtement architectural et décoratif, des références à d'autres univers artistiques - du textile (fig. 2) et de la joaillerie à l'art du métal et du verre, en passant par l'ébénisterie et l'architecture, ou la gravure et la peinture. En intégrant le domaine des arts décoratifs, les compositions d'azulejos, ou compositions azulejares, témoignent des interactions visuelles et matérielles provenant du contact entre plusieurs cultures et civilisations ${ }^{8}$. C'est ainsi que l'on peut percevoir, en se penchant sur son caractère hybride et multifacette, fruit de divers processus d'échanges, d'adaptations continues et de moyens de traduction visuelle et matérielle, quelques-unes des dynamiques les plus originales et intéressantes de l'art portugais.

[Alexandra Curvelo] 


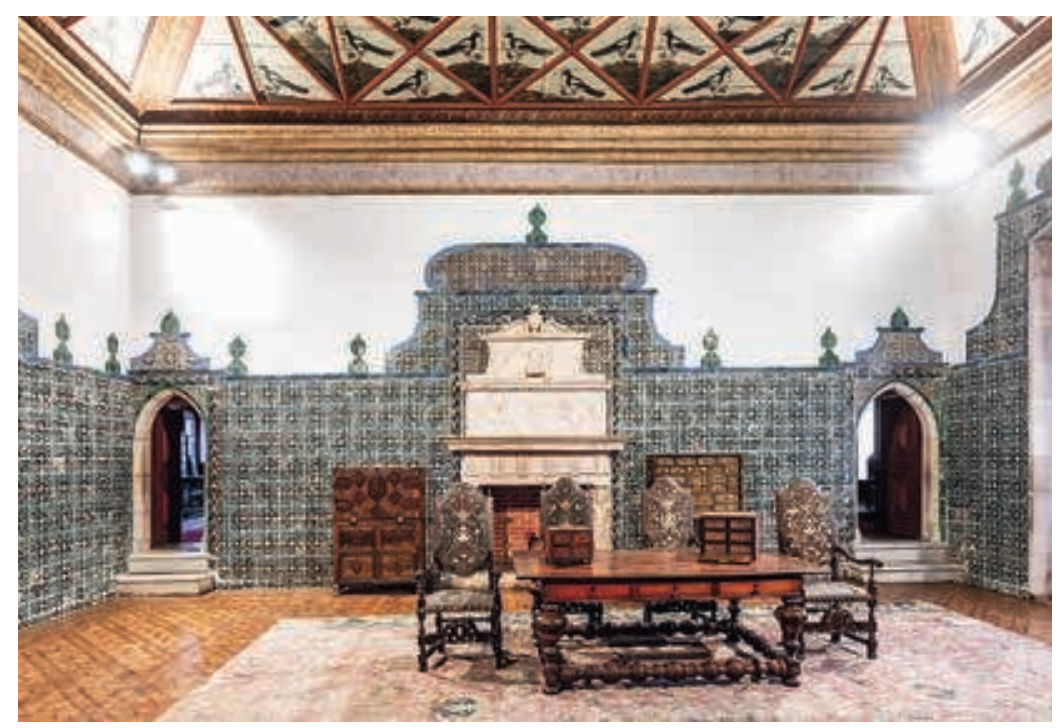

3. Intérieur du Palácio - Alexandra Curvelo. À partir de quand, et comment, peut-on parler da Vila de Sintra. d'azulejo portugais?

- Rosário Salema de Carvalho. On peut en parler à partir de la fin du XVe et du début du XVI ${ }^{e}$ siècle. Mais quelques explications sont nécessaires, notamment parce que la question des origines est complexe et n'est pas exempte de controverses. Si on l'aborde du point de vue de la production, en entendant par « azulejo portugais » les premières expériences ou tentatives de fabrication au Portugal, on entre dans un sujet sur lequel les spécialistes de l'étude de l'azulejaria dite mudéjar travaillent depuis longtemps : celui de l'existence, même résiduelle ou expérimentale, d'une telle production sur le territoire national. Sans vouloir alimenter un débat qui remonte au tournant du XXe siècle et que les découvertes archéologiques des dernières décennies ont nourri ${ }^{9}$, mais en partant du principe que cette hypothèse de travail est parfaitement valable, on pourrait dater l'azulejo portugais de la fin du XVe ou du début du XVI siècle.

D'un autre côté, si l'on considère qu'« azulejo portugais » désigne un concept, ou comme le disait Reynaldo dos Santos ${ }^{10}$ (parmi tant d'autres), une " vision monumentale de son application », on entre dans le domaine d'un courant historiographique qui défend, depuis la seconde moitié du XIXe siècle, son originalité. Et cette singularité ne réside ni dans la technique ni dans les motifs peints, mais surtout dans le «sens décoratif et ornemental » de son usage, raison pour laquelle des exemplaires importés peuvent acquérir un caractère "portugais » selon la façon dont ils sont employés en architecture. Dans ce cas, on peut aussi le dater de la fin du XVe siècle.

- Alexandre Pais. Si l'on envisage cette question du point de vue de l'interprétation du revêtement azulejar au Portugal, on doit remonter aux importations de Séville, à la fin du XV siècle. L'exemple le plus représentatif, bien qu'il n'en reste que des traces, est peut-être celui de la Sé Velha de Coimbra, I'ancienne cathédrale ${ }^{11}$. Son originalité contraste avec la méthode hispanique et provient d'une utilisation plus libre de l'azulejo, qui s'affranchit du modèle géométrique. La norme, en Espagne, ce sont des compositions répétitives qui reproduisent les motifs du modèle pour créer des compositions géométriques disposées et encadrées 
par des lignes droites. Au Portugal, l'azulejo est plutôt disposé de façon à former des motifs. Cet effet est renforcé par le contraste entre les différentes compositions et par la prédominance des couleurs de chaque azulejo, qui créent des dissemblances dynamiques et, finalement, des structures illusoires et scénographiques. Lorsqu'ils sont disposés de façon canonique, qu'ils composent un espace rempli du même type de motifs, les limites de la composition constituent une scénographie. Les salles du Palácio da Vila de Sintra ${ }^{12}$ (fig. 3) en sont l'exemple parfait : les extrémités des surfaces azulejares évoquent des espaces murés, qui dématérialisent en partie les limites architecturales.

Mais si la question renvoie à la production d'azulejos au Portugal et aux caractéristiques qui définissent sa spécificité artistique, alors il faut se concentrer sur la seconde moitié du XVI ${ }^{e}$ siècle. À Lisbonne, les ensembles de l'église de Graça (fig. 4 ; vers 1670), du flamand João de Góis, ou de la chapelle São Roque (1584), de Francisco de Matos, reflètent des aspects propres à l'azulejaria nationale : un discours de nature symbolique et non pas simplement décoratif, en lien avec l'architecture et avec sa finalité ; la dimension illusoire de la représentation ; la spécificité des compositions qui perdent leurs sens lorsqu'elles sont déplacées ou décontextualisées.

- Alexandra Curvelo. Le grand public associe surtout la production azulejar portugaise au XVIII ${ }^{\text {e }}$ siècle et à l'azulejo bleu et blanc. Cette production apparaît à la fin du XVII ${ }^{e}$ siècle et découle en partie des commandes passées à la Hollande, qui ont eu un impact sur le goût des clientèles nationales et dont les peintures bleues sur blanc évoquent l'influence de la porcelaine chinoise. Cette nouvelle esthétique, l'une des « images de marque » de l'azulejo portugais, a assurément modifié la production azulejar au Portugal et ouvert, dans le premier quart du XVIII siècle, ce que I'historiographie appelle le «Cycle des maîtres ${ }^{13}$ » (fig. 5), avec l'apparition des premières compositions signées.

Pourtant les recherches de ces vingt dernières années, auxquelles le Museu nacional do azulejo (MNAZ) a contribué de façon décisive, notamment à travers plusieurs expositions temporaires et la publication de catalogues ${ }^{14}$, ont révélé I'importance et la singularité de l'azulejo du XVII' siècle. D'ailleurs, il est intéressant d'observer comment
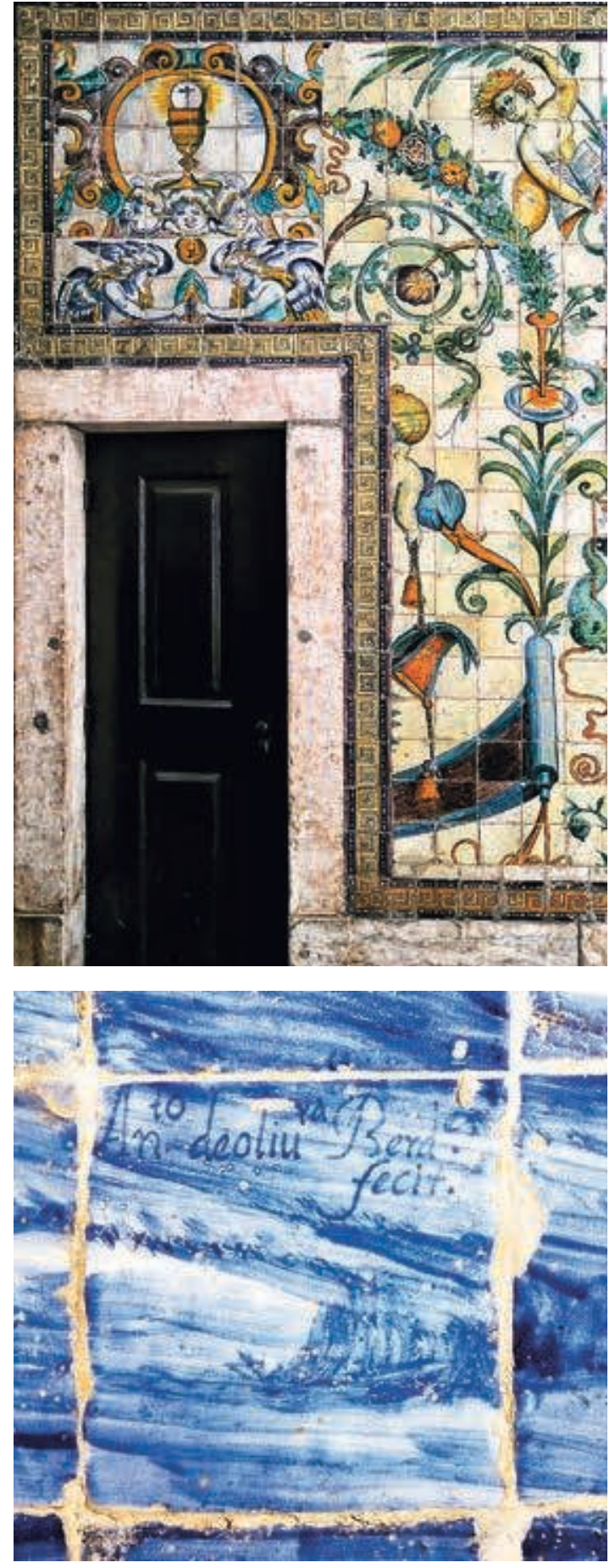

4. Décor intérieur (détail) de l'église de Graça, Lisbonne.

5. António de Oliveira Bernardes (1662-1732), détail d'un panneau du revêtement de l'ancienne chapelle do Bispo portant la signature de l'artiste, actuelle salle à manger du Convento de São Paulo Hotel-Rural | Museu, Aldeia da Serra d'Ossa, Redondo. 

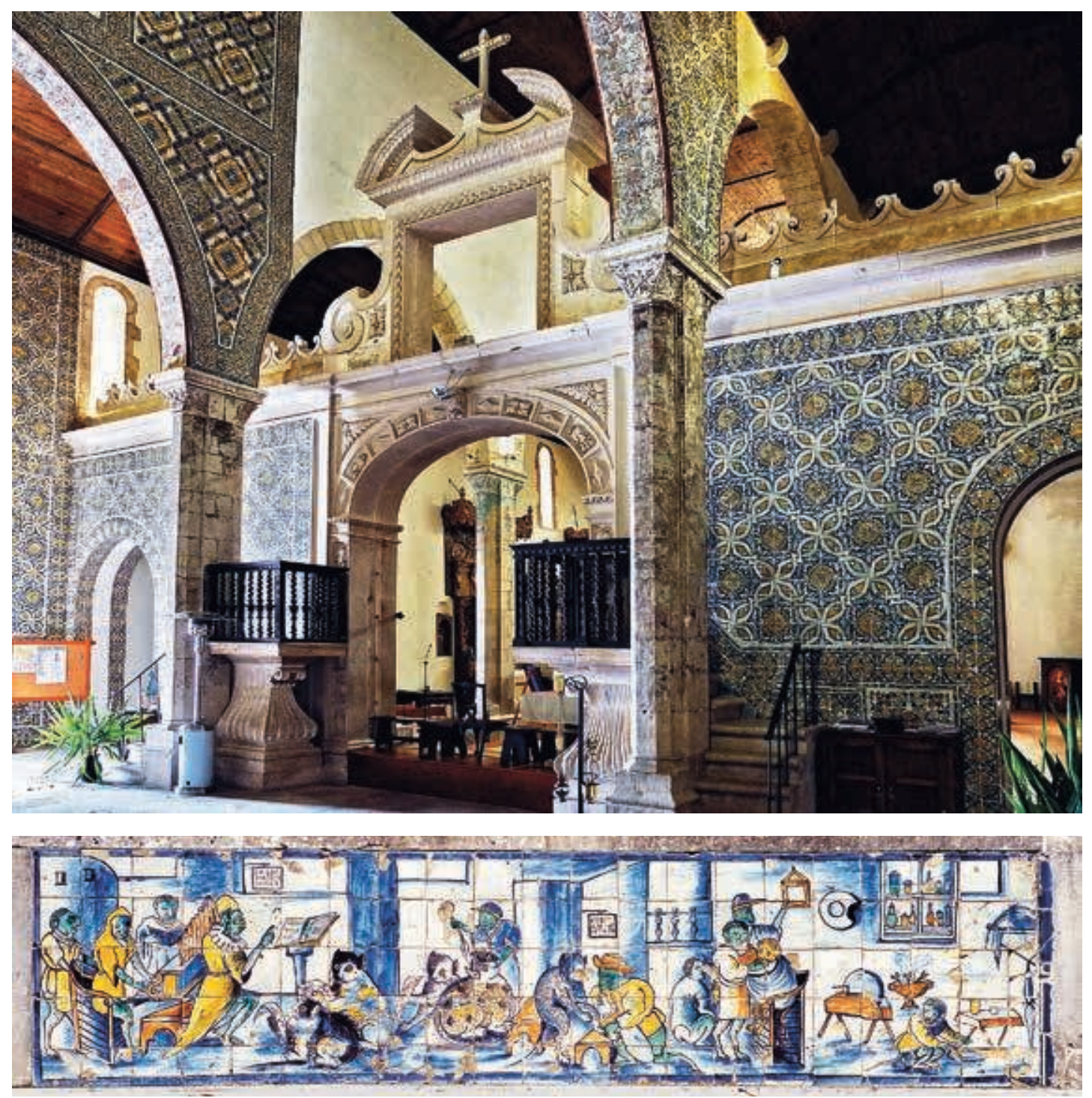

6. Revêtement azulejar, XVII siècle, azulejos à motifs et " en tapis ", église du monastère Santa Maria de Almoster, Santarém.

7. Azulejos figuratifs, XVII siècle, palais Fronteira, Lisbonne (Benfica) ce siècle, dont l'image a longtemps été assombrie par contraste avec les $\mathrm{XVI}^{\mathrm{e}}$ et XVIII siècles, presque comme s'il s'agissait d'un "Moyen Âge » entre deux moments brillants, a été revalorisé. Dans le cas de l'art et de la culture portugais, quelles sont les grandes lignes de cette nouvelle approche?

- Alexandre Pais. Je crois que l'azulejo du XVII siècle est peut-être le meilleur marqueur des convulsions de son temps, offrant les indices les plus révélateurs. Pendant la période de I'Union ibérique (1580-1640), la production d'azulejos a fleuri au Portugal, ce qui indique un éloignement de la production hispanique, et surtout de ses principaux concurrents : les poteries de Talavera. Les ateliers de poterie portugais ont œuvré à créer une azulejaria aux motifs majoritairement géométriques et de plus en plus complexe (fig. 6); dans la profusion de ses combinaisons on identifie encore aujourd'hui des nouveautés, malgré 
un inventaire systématique mené, surtout, par João Miguel dos Santos Simões ${ }^{15}$. Dans la production céramique nationale, comme dans d'autres domaines artistiques, on voit apparaître une résistance qui se traduit par la création d'une identité s'affirmant face à une Union ibérique peu à peu contestée par une faction de la société portugaise. On peut aussi observer cette dimension in situ dans des lieux comme le palais Fronteira, à Benfica (fig. 7), ou sur des panneaux d'azulejos qui sont aujourd'hui, décontextualisés, conservés dans les collections du MNAZ ou du musée Berardo, à Estremoz.

Après la restauration de l'indépendance, l'azulejaria témoigne du manque de financement et de matières premières dans le Portugal de l'époque. On remarque, par exemple,

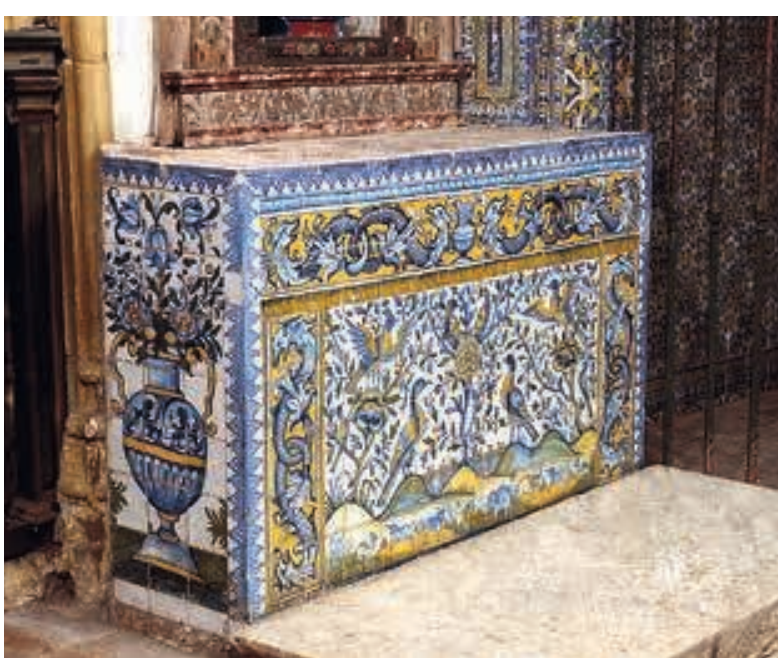

8. Antependium, $\mathrm{XVII}$ siècle, Convento da Esperança, Alcáçovas (Alentejo). l'utilisation de matériaux moins onéreux et la quasi-disparition du bleu d'oxyde de cobalt, le pigment céramique le plus onéreux à l'époque, qui y est remplacé par des oxydes plus accessibles dont l'extraction est probablement locale, mais les résultats moins heureux. L'emploi de l'oxyde de cobalt, qui redevient notable dans la production de la fin de ce siècle et surtout du siècle suivant, correspond de fait à la période dorée de la découverte des mines au Brésil. Elle ouvre un nouveau moment de l'histoire du Portugal, dont le luxe et l'ostentation sont comparables, bien que différemment, à ceux qui marquent la première moitié du XVI $I^{\mathrm{e}}$ siècle. Autrement dit, il n'y a pas un XVII siècle dans l'azulejaria, mais bien des moments qui suivent le cours de l'histoire.

Les recherches les plus récentes, grâce à des méthodes d'examen et d'analyse en laboratoire, ont aussi souligné ces aspects distinctifs. Les résultats obtenus montrent une rupture avec les modes de production du XVI siècle et l'apparition de procédés dont on pourrait quasiment dire qu'ils définissent l'azulejaria des deux siècles suivants. Le MNAZ a joué un rôle fondamental dans ces études, à travers plusieurs expositions temporaires et la publication de catalogues $^{16}$, et contribué à la reconnaissance de la singularité de l'azulejo du XVII siècle, longtemps éclipsé par la fulgurance de cette expression artistique aux XVI et XVIII siècles.

- Rosário Salema de Carvalho. On peut en effet affirmer que le XVII ${ }^{\mathrm{e}}$ siècle est l'une des périodes les plus riches de l'histoire de l'azulejaria portugaise. La diversité des propositions observées y est remarquable : des azulejos enxaquetados (" en échiquier ») aux revêtements d'antependiums (fig. 8), en passant par des combinaisons décoratives aux couleurs de plus en plus éclatantes, plus tard remplacées par des compositions figuratives et narratives en bleu et blanc, qui lanceront une mode pratiquée pendant de longues décennies... Et en « toile de fond » subsistent les azulejos géométriques, dont la quasi-omniprésence est double : soit ils suivent les modes, en termes de motifs et de tonalités, soit ils reprennent des modèles déjà existants, les faisant perdurer dans le temps.

L'exposition organisée par le MNAZ17 en 2012 a grandement contribué à porter un nouveau regard sur ce siècle. Son catalogue, qui en est la trace concrète, est le lieu d'une lecture pluridisciplinaire d'une pertinence considérable : il comporte des études portant sur d'autres arts connexes, comme l'orfèvrerie ou le textile, et fait la part belle à la recherche 


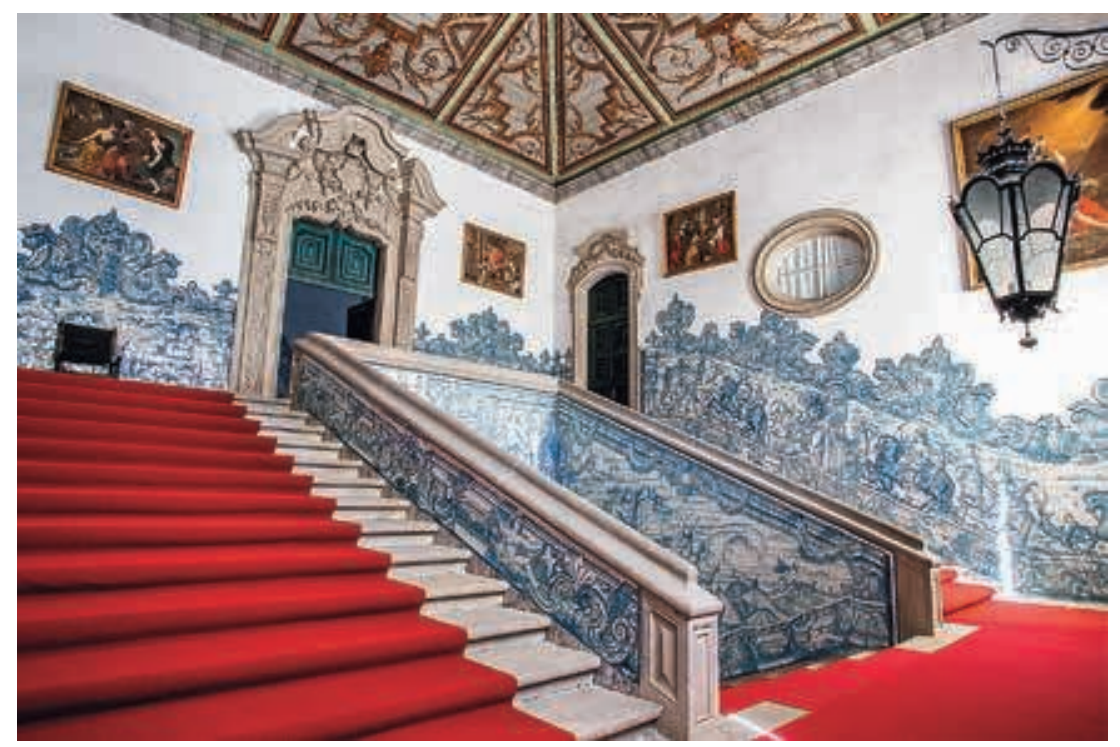

9. Grand escalier du palais Anadia, second quart

matérielle, sous la forme de l'analyse morphologique du XVIII siècle, Mangualde (Viseu).

et chimique qui a fourni des données extrêmement importantes, pour ce siècle comme pour d'autres périodes. C'est dans ce contexte qu'a été lancé un nouveau projet d'études des motifs ${ }^{18}$, qui vise à actualiser le premier catalogue de motifs du XVII ${ }^{\mathrm{e}}$ siècle publié en 1971 par João Miguel dos Santos Simões.

Cependant il me semble important de souligner que ce nouveau regard s'attache aussi depuis quelques années à d'autres siècles. On assiste en effet à un renouvellement thématique et méthodologique notable, conduit par une jeune génération de chercheurs (le mot « jeune » ne concernant pas seulement l'âge bien sûr). M'en tenant aux thèmes uniquement, j'évoquerais l'azulejaria produite à Coimbra (fig. 9), étudiée par Diana Gonçalves dos Santos ${ }^{19}$, le « retour à la figuration ", qui désigne dans les travaux de Tiago Borges Lourenço ${ }^{20}$ I'azulejaria de la transition vers le XXe siècle, ou l'azulejo de façade (fig. 10), qui était encore très peu valorisé et étudié il y a une dizaine d'années, excepté par Ana Margarida Portela ${ }^{21}$ ou Francisco Queiroz ${ }^{22}$.

- Alexandra Curvelo. Une question importante pour l'étude de l'azulejo portugais a trait à sa matérialité (I'azulejo en tant que culture matérielle ${ }^{23}$ et à son processus de conception et de fabrication - des sujets auxquels vous avez tous deux consacré une partie de vos recherches ${ }^{24}$. Concrètement, il s'agit de savoir qui commandait les compositions azulejares, surtout celles de grandes dimensions ; qui définissait les thèmes; qui choisissait les matrices (peintes, gravées ou autre); qui fournissait la matière première ; où se trouvaient les fours (fig. 11); qui mesurait les murs et adaptait l'échelle des compositions à leur lieu de destination ; qui peignait les azulejos avant la cuisson ; qui les posait et, aussi, comme l'a écrit João Miguel Simões, « qui orchestrait tout ce processus de façon chirurgicale au point que des ateliers lisboètes produisaient des panneaux immenses qui étaient posés à des centaines ou à des milliers de kilomètres de distance sans la moindre faille, comme s'ils avaient été naturellement peints sur place ${ }^{25}$ ? »Comment a-t-il été possible de répondre à certaines de ces questions, et dans quelles mesures les informations recueillies ont-elles changé la façon d'écrire sur l'azulejo portugais? 


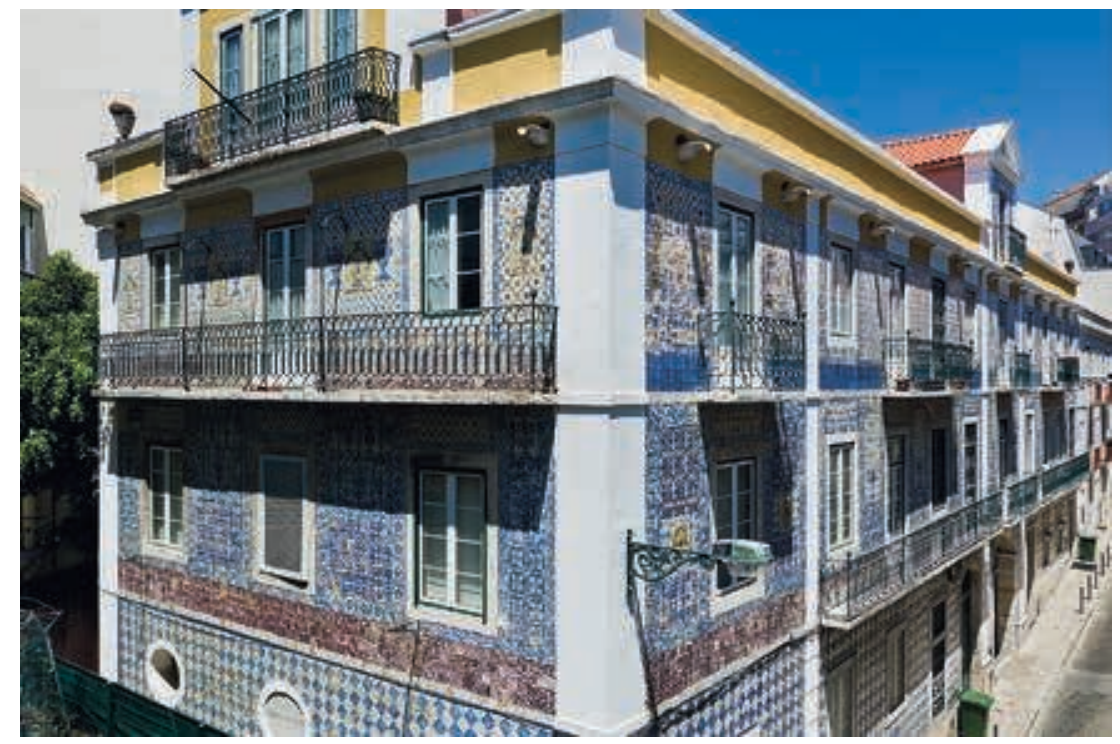

- Rosário Salema de Carvalho. Nos recherches ont non seulement fourni des réponses à certaines de ces questions, en apportant de nouveaux éléments ou en corroborant la perspective de travaux de recherches antérieurs, mais elles ont aussi soulevé de nouvelles interrogations. La vision que l'on a aujourd'hui de l'azulejo portugais n'est pas fondamentalement différente, elle est surtout plus approfondie et plus rigoureuse sous de multiples aspects.

Bien que des auteurs comme Vergílio Correia ${ }^{26}$ ou Santos Simões ${ }^{27}$ aient apporté une contribution décisive à ces problématiques, les dynamiques entre les acteurs impliqués dans les processus de commande et d'application d'un revêtement céramique et leur rôle effectif dans ce cadre complexe n'ont pas toujours été bien comprises ou interprétées. En cherchant à inverser cette situation, la Rede de investigação em azulejo, avec le MNAZ et le Centro de história de arte e investigação artística (CHAIA, Universidade de Évora) ont organisé, en 2017, le séminaire "Quem faz o quê: processos criativos em azulejo » ("Qui fait quoi : processus créatifs dans l'azulejo »). En parallèle, les auteurs ont été invités à contribuer à l'Az Infinitum - Sistema de referência e indexação de azulejo ("Système de référencement et d'indexation de l'azulejo ») en remplissant une fiche pour chaque intervenant dans la création et la mise en œuvre des revêtements ${ }^{28}$. Cette expérience a montré qu'il était nécessaire de continuer à recourir aux pratiques collaboratives et aux nouveaux outils technologiques, qui permettent de cartographier les réseaux complexes de relations et de les visualiser de façon immédiate et très efficace.

Les études dans ce domaine avancent grâce à la recherche dans les archives, et je voudrais signaler ici l'importante contribution de Celso Mangucci $\mathrm{i}^{29}$ concernant le XVIII e siècle ou bien la chance que j'ai eue moi-même de découvrir et de pouvoir analyser le premier Regimento do ofício de ladrilhador (" Règlement du métier de carreleur »), qui date de 1608 et se trouve dans une collection privée ${ }^{30}$. Mais s'il existe encore un large champ à explorer, et les approches monographiques en ce sens continuent de manquer, il est aussi vrai que les chercheurs doivent actualiser leurs méthodes, en utilisant les technologies aujourd'hui à leur disposition, sans hésiter à employer pour l'étude de l'azulejo des outils novateurs, voire en se risquant aux approches de la vision par ordinateur ou de l'intelligence artificielle, comme nous essayons de le faire au sein de la Rede de investigação em azulejo. 
- Alexandre Pais. Au-delà de la contribution des recherches historiographique et documentaire, d'autres disciplines comme la chimie, la géologie et la physique nous ont aidé à comprendre, entre autres, les contextes de production. L'intervention du Laboratório Hércules (Universidade de Évora), de plusieurs laboratoires de l'Universidade Nova de Lisboa, et surtout du Laboratório nacional de engenharia civil (LNEC) a permis d'obtenir des informations sur l'origine des matières premières, sur les structures qui fabriquaient les azulejos et même sur le lieu de production des ensembles conservés sur le territoire national ${ }^{31}$. Les chercheurs d'un projet pionnier, basé au LNEC, ont pu déterminer avec rigueur les traits distinguant l'azulejo portugais des azulejos flamand et hollandais, et ils cherchent actuellement à établir des différences avec la production espagnole ${ }^{32}$. En parallèle, des études sont menées pour définir des procédés de cuisson qui permettront, à moyen terme, d'identifier les fournées d'origine de nombreux centres azulejares du territoire portugais, et donc de savoir un peu mieux qui fabriquait les azulejos et, nous l'espérons, qui les peignait. Le contact avec des institutions néerlandaises et espagnoles a placé ces perspectives sur le devant de la scène et engendré un intérêt croissant de ces domaines du savoir pour la recherche en azulejo, contribuant ainsi à une avancée significative des connaissances et à l'obtention de réponses plus claires sur cette production.

- Alexandra Curvelo. Vos travaux respectifs portent sur la production céramique portugaise,

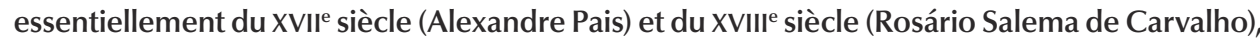
et vous y envisagez l'azulejo non pas comme objet principal, mais comme point central de la recherche. Grâce à vos travaux et aux postes que vous occupez, Alexandre Pais en tant que conservateur du Museu nacional do azulejo, après un passage par I'Instituto português de conservação e restauro, et Rosário Salema de Carvalho en tant que coordinatrice de I'Az - Rede de investigação em azulejo (" réseau de recherche sur l'azulejo ") ${ }^{33}$, vous faites le lien entre le milieu universitaire et les institutions culturelles, surtout muséologiques, mais aussi avec des programmes spécifiques pour la sauvegarde du patrimoine azulejar ${ }^{34}$. Comment s'exerce ce travail en réseau et quels résultats pratiques a-t-il produit, notamment en termes d'impact sur la société ?

- Alexandre Pais. Au cours de quinze dernières années, l'intérêt pour l'azulejo, en tant que matériau et thématique, s'est accru, tout comme la reconnaissance de son importance. Plusieurs facteurs y ont contribué : la création de l'Az - Rede de investigação em azulejo, basée à la faculté de Lettres de I'Universidade de Lisboa et coordonnée par Rosário Salema de Carvalho ; I'ouverture du MNAZ à des stages pratiques d'inventaire et d'études de son fonds azulejar ; le dispositif SOS Azulejo du Museu da polícia judiciária, une initiative fondamentale pour assurer la sauvegarde des azulejos, l'éducation patrimoniale (auprès des écoles) et la sensibilisation du public à sa responsabilité dans la préservation des azulejos; et puis la constitution, par les mairies de Porto, d'Ovar et d'Aveiro, de bancos de azulejos (littéralement, "banques d'azulejos »), qui permettent de restaurer des façades (fig. 12) avec des pièces originales provenant d'édifices en voie de démolition ou de modification.

La création du projet AzLab ${ }^{35}$, dont les conférences mensuelles sur des thèmes liés à l'azulejo sont ouvertes au public, et le lancement d'Az Infinitum, une base de données en ligne visant l'identification et la connaissance de ce patrimoine, ont aussi joué un rôle dans le développement et la diffusion de cette culture matérielle portugaise. Le MNAZ n’avait jamais reçu autant de demandes de stages de la part d'étudiants en histoire de l'art, en architecture, aux beaux-arts, en conservation et restauration, en muséologie, etc. qu'aujourd'hui.

11. Gabriel del Barco (attr.), Grand Panorama de Lisbonne avant le séisme de 1755, détail des ateliers de poterie, vers 1700 céramique émaillée, majolique, Lisbonne, Museu nacional do azulejo, Inv. $1 \mathrm{Az}$. 

Un autre aspect notable du travail mené ces dix dernières années est qu'il est désormais possible de développer des projets de recherche solidement axés sur la pratique, permettant le contact et la compréhension des objets inter- et/ou multidisciplinaires. Pour toutes les personnes impliquées, ce travail en réseau renforce le projet de proposer à I'Unesco le classement de l'azulejo portugais dans la liste du patrimoine mondial.

- Rosário Salema de Carvalho. Aujourd'hui, plus que jamais, le caractère patrimonial de l'azulejo est reconnu, par les étrangers mais aussi et surtout par les Portugais, et cela en dit long sur le travail que les différentes institutions ont mené pour son étude et sa sauvegarde. Le problème, c'est qu'il y a encore tant à faire ! Notre principale mission est l'étude et la recherche, et de nombreuses institutions nous contactent parce qu'elles veulent inventorier, documenter et mieux connaître leur patrimoine. D'autres chercheurs trouvent dans la Rede de investigação em azulejo un espace de débat privilégié et les infrastructures nécessaires à la poursuite de leurs travaux. Nous communiquons avec le grand public en ouvrant nos actions à tous, des séminaires spécialisés à la publication des résultats d'études, que ce soit dans des articles scientifiques ou dans des ouvrages plus abordables incluant la participation des principaux chercheurs en la matière, ou encore par des moyens plus accessibles, en publiant régulièrement sur nos réseaux sociaux ${ }^{36}$. Dans ces séminaires du projet AzLab nous tâchons de donner la parole aux chercheurs, des plus consacrés comme José Meco ou Vitor Serrão, dont nous reconnaissons le rôle considérable qu'ils continuent à jouer, aux plus jeunes qui font leurs premiers pas dans ce domaine, mais aussi aux artistes, aux restaurateurs, aux techniciens du patrimoine travaillant dans les institutions les

12. Restauration d'une façade d'azulejos à Ovar.

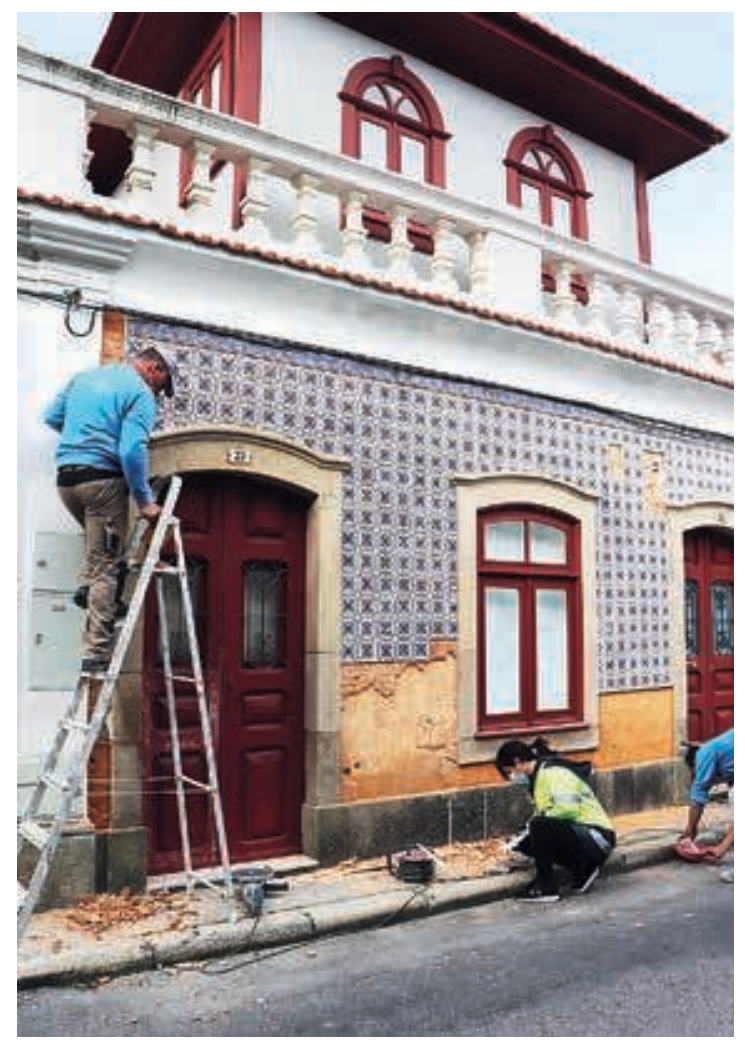
plus diverses, aux conservateurs de musée, aux collectionneurs, parmi d'autres, qui présentent des regards croisés depuis différentes disciplines scientifiques. Plus récemment, ces séminaires ont acquis une dimension internationale, d'une part grâce aux invités étrangers qui permettent d'élargir considérablement ce réseau de connaissances, d'autre part grâce à la transmission des séances en ligne et à la mise à disposition des vidéos sur notre chaîne YouTube.

Dans toute cette dynamique, l'un des points les plus préoccupants à nos yeux est la préservation des revêtements azulejares dans les lieux où ils ont été posés (fig. 13). L'azulejo est un patrimoine intégré et il doit le rester. Lorsque l'on retire un ensemble d'une façade ou d'un intérieur, même s'il est composé d'azulejos à motifs, on lui ôte tout le contexte qui est essentiel à sa compréhension globale. C'est pourquoi notre relation avec les musées se fonde beaucoup sur l'étude des provenances, entendues ici comme lieu d'origine : nous cherchons à réactiver ces liens si souvent perdus, surtout dans le cas d'intégrations anciennes. S'il est vrai que sans les musées (publics ou privés) nous n'aurions aujourd'hui pas accès à une part considérable de ce patrimoine, il est essentiel 


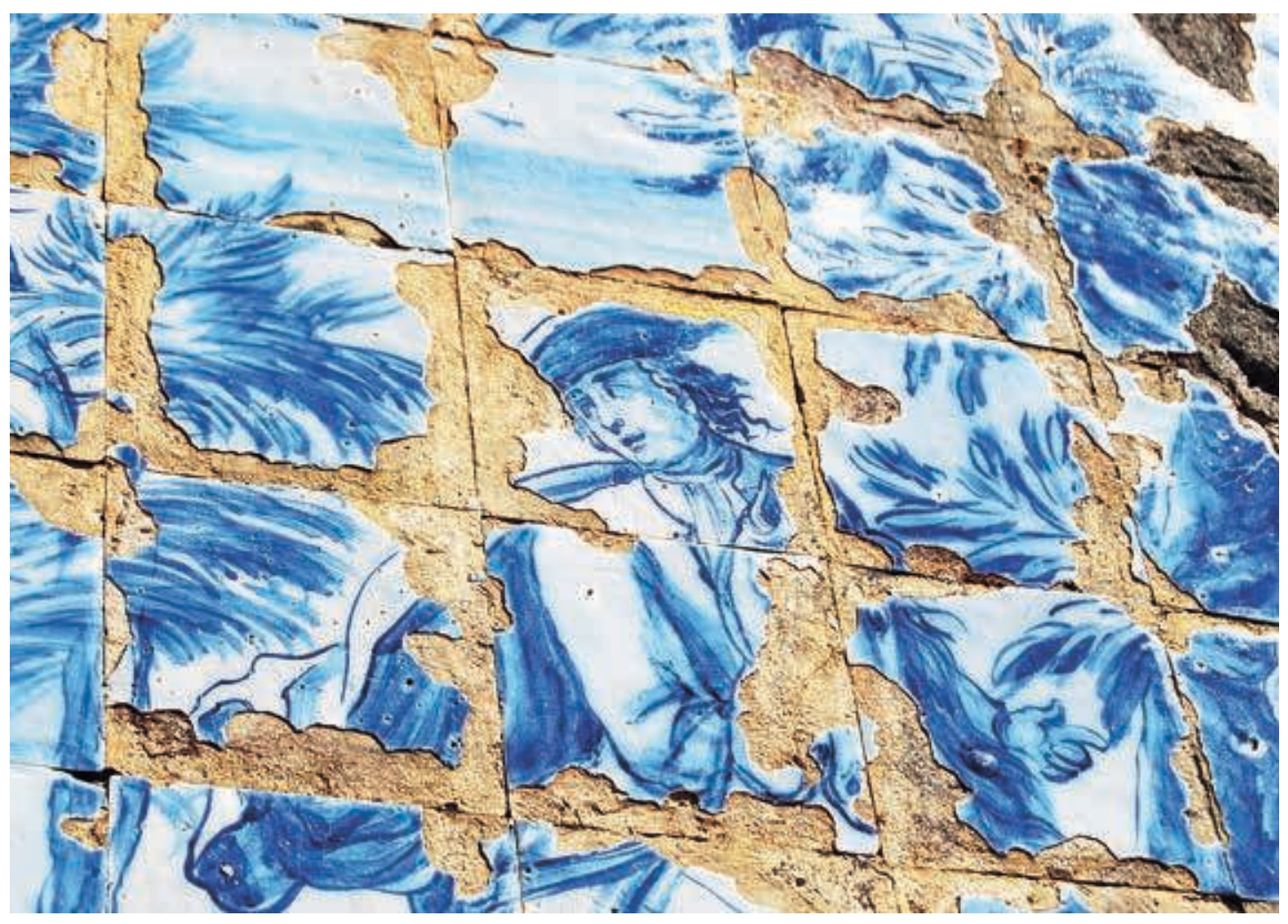

que ceux-ci puissent adopter des stratégies pour montrer 13. Azulejos en situation de risque, à leurs visiteurs que l'azulejo « vit » dans l'architecture et Lisbonne. dans la relation qu'il établit avec d'autres manifestations artistiques présentes dans le même espace.

Cette préservation relève d'ailleurs d'une sensibili-

sation très large qui devrait être faite au niveau national. Il existe des cas absolument exceptionnels, comme celui de la mairie d'Ovar, où l'on réalise des actions « au porteà-porte ». II en faudrait plus! D'un autre côté, l'idée selon laquelle on ne protège que ce que l'on connaît reste valable, et c'est une mission qui est loin d'être achevée, malgré les nombreux progrès accomplis en termes d'inventaire. Au sein de la Rede de investigação em azulejo, nous nous efforçons de soutenir les initiatives d'inventaire, et nous avons les moyens de le faire, car nous pouvons constituer des équipes et structurer des projets. L'Az Infinitum, notre projet ressource, justement développé avec le MNAZ pour mettre en pratique la stratégie de recréation de liens que j'ai évoquée plus haut, a été pensé pour inventorier le patrimoine azulejar et surtout pour le documenter plus largement. Et les stratégies futures passent par l'articulation numérique d'inventaires divers.

Concernant la sauvegarde, il est important de mentionner le dispositif SOS azulejo, qui joue un rôle important dans la promotion des cadres légaux, dans la prévention du vol et du vandalisme, et dans la dynamisation de prix annuels et de l'Ação Escola (" action école »), d'excellents outils de valorisation et de divulgation.

- Alexandra Curvelo. Dans quelle mesure associez-vous, ou non, les concepts de patrimoine, de mémoire et d'identité à l'azulejo portugais, et ce faisant quelles questions en découlent, surtout dans une perspective future? 


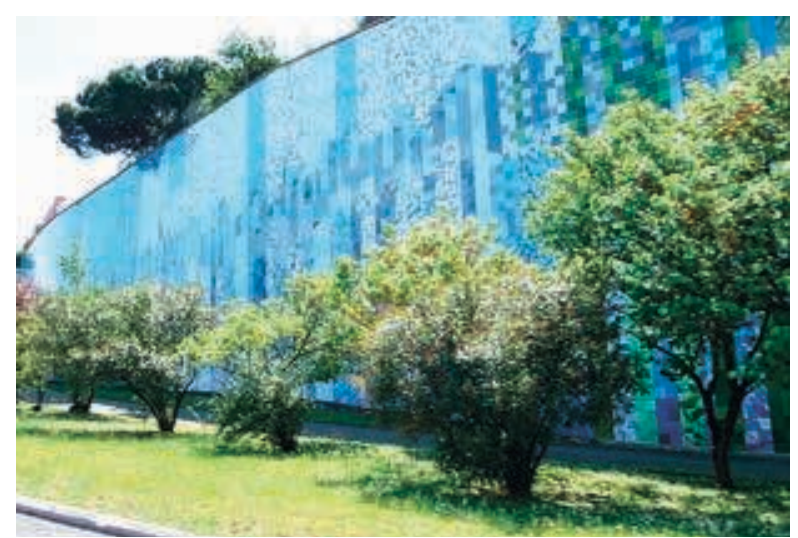

14. João Abel Manta, revêtement mural en azulejos, 1970 (création) - 1982 (pose des séries azulejares), Lisbonne, avenida Calouste Gulbenkian.
- Rosário Salema de Carvalho. Il est impossible pour nous de ne pas associer ces concepts. Mais il est également essentiel d'avoir conscience de ce qu'ils impliquent et de saisir ce qui est le plus important : souligner les différences et mettre l'accent sur une soi-disant identité, qui nous isole, ou bien valoriser ce qui nous unit et comprendre l'azulejo dans un contexte plus vaste, européen, et dans une certaine mesure à une échelle mondiale ? L'azulejo est utilisé au Portugal depuis la fin du XVe siècle, et ce sans interruption; les créations contemporaines sont très révélatrices de sa vitalité et offrent aussi de bonnes perspectives pour le futur. En fait, depuis la seconde moitié du XX⿳亠丷厂犬 siècle, les formes et les limites de l'azulejo sont en permanence mises en question - y compris dans ces lieux d'application - et les propositions constamment renouvelées, ce qui est d'ailleurs vrai tout au long de l'histoire de l'azulejaria. Il me semble donc essentiel, plutôt que de débattre de ces concepts, d'essayer d'envisager l'azulejo en intégrant la pluralité des regards qu'il permet, en l'abordant via l'histoire de l'art, mais aussi par le biais d'autres disciplines, comme la chimie ou l'anthropologie, la vision par ordinateur ou la musique, en passant par les mathématiques ou même la médecine. L'azulejo, peut-être par sa nature de patrimoine ou la force de sa mémoire, ne s'épuise pas dans une vision unique : il renferme au contraire des approches multiples, singulières ou globales.

- Alexandre Pais. La notion de patrimoine est indiscutable concernant la culture matérielle de l'azulejo au Portugal. Employé depuis plus de cinq cents ans sans interruption (fig. 14), encore fabriqué et utilisé de nos jours, relevant de conceptions et de contextes divers, il est une expression marquante et vivace de l'art portugais.

Concernant la mémoire, on peut sentir et interpréter dans l'azulejaria nombre d'éléments qui, associés à la mémoire, renvoient à sa création, à sa fabrication, à sa représentation, son utilisation et sa valorisation. On trouve ainsi le sentiment de la découverte dans les ensembles expérimentaux du XVI $\mathrm{X}^{\mathrm{e}}$ siècle ; la recherche d'autonomie face à l'union avec la Couronne espagnole puis les angoisses dans la période de l'indépendance qui lui succède, au XVII siècle ; la splendeur et la construction narrative marquant le faste et l'abondance des ressources au XVIII ${ }^{e}$ siècle ainsi que l'ascension, au XIX ${ }^{e}$ siècle, d'une bourgeoisie qui recherche l'ostentation; la formation d'un discours d'État, la construction de l'engagement dans des causes sociales et de nouvelles façons de communiquer au XXe siècle. Tout cela se reflète dans les productions de ces époques et fait écho à notre compréhension présente de I'histoire. La production actuelle peut aussi être envisagée comme une mémoire, issue des archives des expressions urbaines de la culture mondialisée du XXI siècle. La mémoire, dans l'azulejaria, c'est aussi sa capacité de réinvention perpétuelle, dont témoignent l'interprétation des motifs, des techniques et des matériaux, employés et perçus de différentes façons selon les moments historiques, dans une pluralité de contextes et de situations et une variété continuelle de créateurs, d'applicateurs et d'utilisateurs.

C'est avec le terme d'identité que tout se complique. Celui-ci peut convoquer des principes nationalistes associables à des propositions relevant de logiques politiques dont j'aimerais 
qu'elles ne contaminent pas cette question fondamentale... Depuis la moitié du XIXe siècle, on constate la reconnaissance internationale du caractère exceptionnel de l'azulejo en contexte portugais. Des recherches nationales et internationales, développées dès cette époque, ont renforcé cet aspect identitaire en lui conférant le sens d'une manifestation singulière lorsqu'on la confronte à d'autres productions. C'est depuis l'étranger vers le Portugal que s'est confirmée la particularité expressive de ce phénomène culturel et artistique national. Preuve en est que le MNAZ, parmi tous les musées affectés à la Direção geral do património cultural (Ministério da Cultura), est celui qui présente le plus haut pourcentage et la plus grande diversité de visiteurs étrangers (près de $82 \%$ contre $53 \%$ pour la seconde structure, selon cette étude). L'azulejo portugais est valorisé à l'échelle internationale comme un élément identitaire de la production artistique nationale - y compris dans l'édition, notamment grâce au travail des éditions Chandeigne ${ }^{37}$. Et je crois que ce facteur est le juge le plus impartial possible, car il est reconnu par celles et ceux qui n'ont aucune implication directe dans la définition interne de cette idée d'identité...

- Alexandra Curvelo. Vous avez tous deux évoqué, et c'est intéressant, l'azulejaria contemporaine, ou plus précisément la présence de l'azulejo, et sa réinvention permanente, dans le panorama de l'art contemporain portugais, ici comme à l'étranger. J'aimerais que vous développiez un peu ce sujet, qui me semble fondamental pour ce débat.

- Rosário Salema de Carvalho. Grâce au recul que nous avons aujourd'hui, nous pouvons relier les différentes composantes de l'azulejaria portugaise à cinq siècles d'histoire et percevoir ses principales tendances. Notre époque ne fera pas exception et l'utilisation actuelle de l'azulejo reflète assurément les dynamiques de l'espace urbain et l'émergence de « nouveaux » espaces ou lieux, tout comme les comportements de leurs habitants. Dans ce contexte, et depuis le milieu du XX $x^{e}$ siècle, le spectre des supports architecturaux traditionnels de l'azulejo s'est élargi, au Portugal comme à l'étranger : il revêt désormais des surfaces aussi variées que des viaducs, des tunnels ou des stations de métro, parmi tant d'autres. Et son langage ne traduit pas seulement l'expression de la personne qui l'a conçu, mais aussi et surtout les différentes façons de penser les espaces et les villes par les artistes, les designers et les architectes.

L'azulejo reste très présent au Portugal. À titre d'exemple, une promenade dans la ville de Lisbonne, en surface comme sous terre, démontre bien son actualité, aussi bien sur des ouvrages publics que sur des façades d'édifices résidentiels ou d'autres bâtiments. Au sein de la Rede de investigação em azulejo, nous nous efforçons de documenter les œuvres contemporaines - on se plaint fréquemment du manque d'informations sur le passé, mais on oublie aussi souvent que l'on peut anticiper ce problème en documentant bien les temps présents. Par ailleurs, la production d'azulejos à motifs se poursuit et l'importance de continuer à collectionner les catalogues de fabricant ${ }^{38}$ a encore été évoquée récemment lors d'une séance de notre séminaire. La documentation, même de ce qui n'est pas valorisé, est essentielle : les façades azulejadas, si critiquées au XIXe siècle et si valorisées aujourd'hui, désormais indissociables du paysage urbain portugais, en sont la preuve. Santos Simões le disait bien : I'azulejo est toujours vivant, I'ancien comme le nouveau, et il nous incombe à tous d'assurer sa pérennité.

- Alexandre Pais. Dans le processus de construction de la tradition azulejar au Portugal, un point semble évident : c'est sa capacité à traverser les siècles. On pourrait penser que cette expression artistique aurait du mal à trouver sa place dans une période de transformations sociales, économiques, esthétiques, si vastes et si complexes. À la fin du XXe siècle, presque toutes 


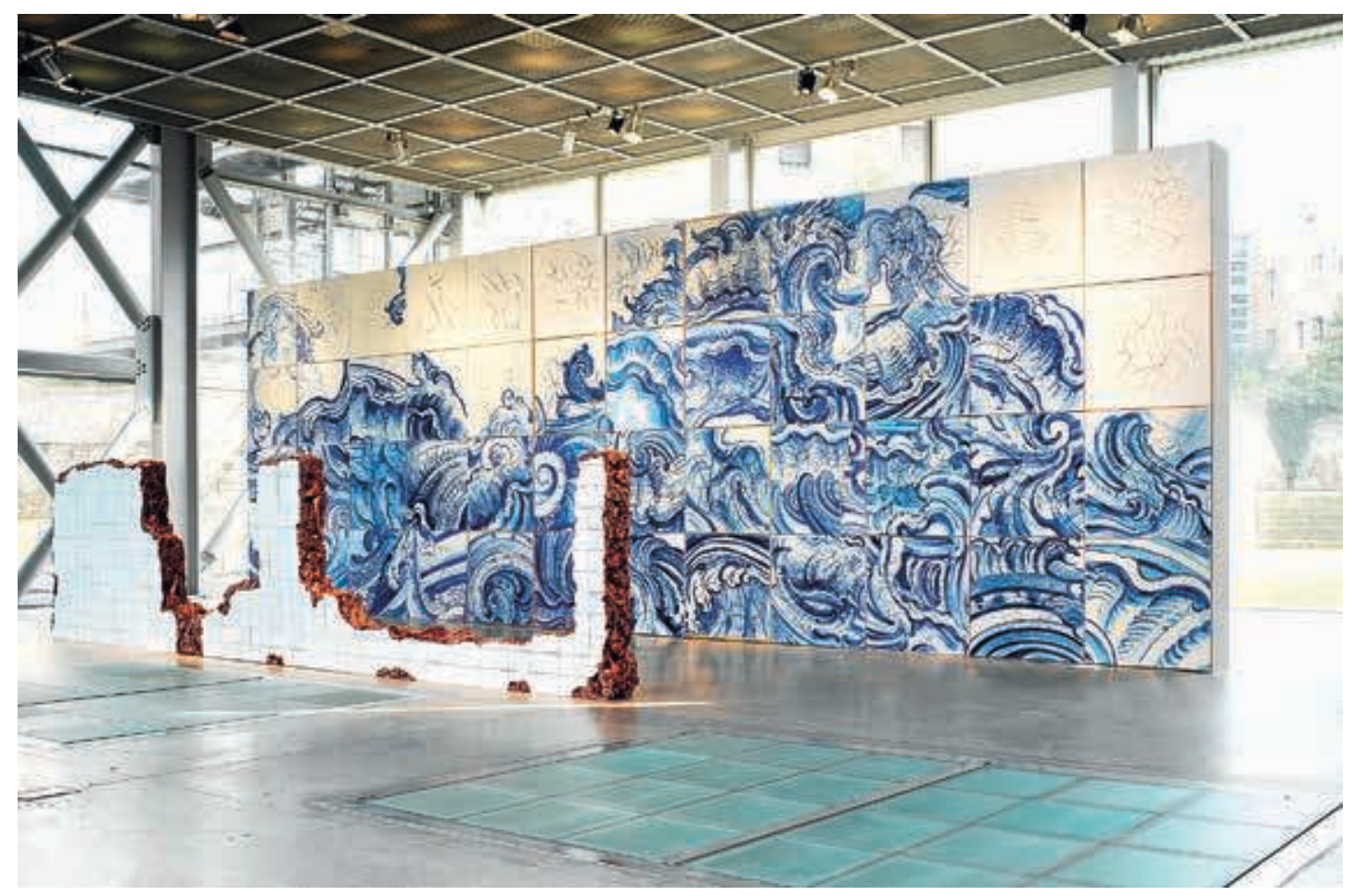

15. Adriana Varejão, Celacanto provoca maremoto [Le cœlacanthe provoque les raz-de-marée], 2004, huile et enduit sur toile, $440 \times 1320 \mathrm{~cm}(4 \times 12$ toiles, $110 \times 110 \mathrm{~cm}$ chacune), dans l'exposition "Chambre d'échos / Câmara de ecos », Paris, Fondation Cartier pour l'art contemporain, 2005. les grandes fabriques traditionnelles d'azulejo du Portugal, des unités de production dont beaucoup avaient été fondées sur le site d'ateliers de poterie dont l'origine remonte aux XVII et XVIII ${ }^{e}$ siècles, ont disparu. Ces fermetures sont le fruit d'une transformation graduelle qui, par ricochet, a réduit à un niveau résiduel les commandes publiques d'azulejos, dont la présence s'était déjà raréfiée dans les espaces portugais de la seconde moitié du XXe siècle. Puis les crises économiques qui ont ponctué les vingt premières années de ce nouveau millénaire sont survenues. Malgré ces conditions adverses, de petites bulles de production existent, qui apportent très souvent de nouveaux langages et de nouvelles méthodes dans la construction esthétique de l'azulejo au Portugal. D'une certaine façon, nous vivons une époque de défis économiques, un contexte proche, pour faire une comparaison rapide, de celui du XVII siècle, après la restauration de l'indépendance du Portugal. Or c'est justement dans le creuset de cette conjoncture qu'est né un moment glorieux de production, un moment qui se révèle aujourd'hui décisif pour définir ce qui est désormais perçu comme l'apogée de l'azulejaria au Portugal : les vastes ensembles en bleu et blanc qui marquent le paysage architectural du Baroque portugais.

Nous assistons bien aujourd'hui à l'introduction de nouveaux langages. Ces esthétiques moins confinées à une production d'auteur, plus ouvertes à un dialogue accessible à différents segments de la société, dont le renouvellement ne permet pas de distinguer clairement l'avenir, nous laissent pressentir un moment de réinvention. Les multiples propositions de l'azulejaria contemporaine semblent marquées par l'intégration de moyens d'expression techniques, comme la photo-impression, qui crée des motifs semblables à la publicité ; la photo-mosaïque, avec la construction d'images de plus grande dimension à partir d'azulejos industriels 
qui ne sont plus produits; la déconstruction d'images en motifs fonctionnant comme des pixels, qui permet d'élaborer des dessins sur des surfaces étendues fonctionnant comme l'écran d'un ordinateur ; la composition d'unités de motifs à partir de graffiti qui paraissent former de loin, une fois organisées, des dessins d'ensemble, mais qui recèlent des surprises pour les yeux plus attentifs ; l'introduction d'éléments en trois dimensions, qu'il s'agisse de morceaux de textile ou de formes géométriques, entraînant la création de plans en relief dans une exubérance jamais vue depuis les expériences du XVI siècle ou les revêtements de façades du XIXe siècle et qui, dans certains cas, entendent recréer du mouvement et accueillent des éléments peints révélant, selon l'angle de vision, la construction de mots. De nos jours, les azulejos ne sont pas tous peints. Certains présentent un motif imprimé, ce qui est parfois vu comme un détournement de la véritable essence de l'azulejaria ; d'autres artistes cherchent à y intégrer des possibilités de changement chromatique selon les changements de lumière et de température ambiante. Quelques expériences tentent aussi d'intégrer et d'amplifier le son dans les surfaces revêtues d'azulejos. On pourrait encore en citer d'autres, mais je pense que les exemples mentionnés montrent bien la dimension expérimentale actuelle. Je crois que tous les moments de transformation portent en eux l'excitation de l'inconnu et de la nouveauté de la création, mais la diversité de propositions à laquelle nous assistons aujourd'hui dans l'azulejaria au Portugal paraît plus viscérale que jamais. Des artistes étrangers demandent à travailler dans les rares unités de fabrication encore actives pour apprendre des techniques, apporter des esthétiques et des langages différents et rapporter dans leurs pays un brin de cette intuition portugaise que l'on retrouve dans le caractère apparemment inépuisable du travail de l'azulejaria. Si nous vivons à tous les niveaux des moments qui nous imposent des défis et qui transforment le monde et notre perception de la place que nous y occupons, il me semble manifeste que l'azulejaria au Portugal cherche à s'y ajuster et à occuper la place qu'elle a toujours eue : celle de témoin de notre sensibilité et de notre capacité d'adaptation au futur.

Traduit du portugais par Élodie Dupau. 


\section{Rosário Salema de Carvalho}

Chercheuse à I'ARTIS - Instituto de história da arte da Faculdade de Letras de I'Universidade de Lisboa, où elle coordonne le groupe de recherche Az - Rede de investigação em azulejo. Elle a coordonné des projets d'études portant sur l'azulejo ainsi que des inventaires, parmi lesquels la base de données pour la documentation de I'azulejo - Az Infinitum - Sistema de referência e indexação de azulejo.

\section{Alexandra Curvelo}

Professeure associée au département d'Histoire de I'art de la Faculdade de Ciências Sociais e Humanas de I'Universidade Nova de Lisboa (NOVA FCSH) ainsi que chercheuse et membre de la direction de I'Instituto de história da arte (IHA). Elle a été conservatrice du Museu nacional do azulejo, technicienne de I'Instituto português de conservação e restauro et du Museu nacional de arte antiga.

\section{Alexandre Pais}

Conservateur du Museu nacional do azulejo, Alexandre Pais a coordonné des projets d'inventaires et de recherches sur l'azulejo, il a été commissaire de plusieurs expositions sur les thèmes de l'azulejaria et de la céramique portugaises. II est le co-rédacteur en chef de la revue Studies in Heritage Glazed Ceramics. On the Origin of Majólica Azulejos Production in Portugal, publiée par le Laboratório nacional de engenharia civil de Lisbonne (LNEC).

\section{NOTES}

1. António Sousa Ribeiro, "Memória », dans Walter Rossa, Margarida Calafate Ribeiro (dir.), Patrimónios de influência portuguesa: modos de olhar, Coimbra, Imprensa da Universidade de Coimbra, 2015, p. 81-94.

2. Roberto Vecchi, "Identidade, herança, pertença », dans Rossa, Ribeiro, 2015, cité n. 1, p. 65-80.

3. Helena Amante, "How Portugal Keeps Alive an Iconic 500-Year-Old Art Form », National Geographic, 8 janvier 2021 [URL : nationalgeographic.com/magazine/2021/02/how-portugal-keeps-alive-an-iconic-500year-old-art-form/].

4. Adalberto Alves, A herança árabe em Portugal, Lisbonne, Clube de Filatelia - CTT Correios, 2001 et Portugal: ecos de um passado árabe, Lisbonne, Instituto Camões, 1999.

5. José Garcia Mercadal, Viajes de extranjeros por España y Portugal, Madrid, Aguilar, 1952 ; Jerónimo Münzer, Viaje por España y Portugal: 1494-1495, Madrid, Ediciones Polifemo, 1991.

6. Voir par exemple José Custódio Vieira da Silva, $O$ tardo-gótico em Portugal. A Arquitectura no Alentejo,
Lisbonne, Livros Horizonte, 1989 et Paços medievais portugueses, Lisbonne, Instituto Português do Património Arquitectónico, 1995 ; ainsi que Pedro Dias, «Arquitectura mudéjar portuguesa: tentativa de sistematização ", Mare Liberum, n 8, 1994, p. 49-89.

7. On peut citer, parmi une vaste bibliographie, les ouvrages de João Miguel dos Santos Simões, Azulejaria Portuguesa no Brasil (1500-1882), Lisbonne, fondation Calouste Gulbenkian, 1965, et de Mário Barata, Azulejos no Brasil: séculos XVII, XVIII e XIX, thèse d'habilitation à diriger des recherches, Rio de Janeiro, Escola de Belas Artes, Universidade Federal do Rio de Janeiro, 1955, ainsi que le numéro de la revue Oceanos, n 36/37, Azulejos. Portugal e o Brasil, octobre 1998 / mars 1999 et enfin deux ouvrages de Dora Alcântara, Azulejos na cultura luso-brasileira, Rio de Janeiro, IPHAN, 1997 et Azulejos portugueses em São Luís do Maranhão, Rio de Janeiro, Fontana, 1980.

8. João Pedro Monteiro, " $A$ Influência oriental na cerâmica portuguesa do século XVII ", dans A influência oriental na cerâmica portuguesa do século XVII, Lisbonne / Milan, Electa, 1994, p. 18-53 ; Alexandra Curvelo, João Pedro Monteiro (dir.), El azulejo en Portugal. Lugar de encuentro de culturas / $O$ azulejo em Portugal. Lugar de encontros de culturas, Cáceres, Fundación Mercedes Calles, 2009.

9. José Gestoso y Pérez, Historia de los barros vidriados sevillanos: desde sus orígenes hasta nuestros días (1903), Seville, Ayuntamiento de Sevilla, Servicio de Publicaciones y Distrito de Triana, 1995 ; Vergílio Correia, O azulejo em Portugal. Uma obra inédita de Vergílio Correia, Lisbonne, Imprensa da Universidade de Lisboa, 2018; Rui André Alves Trindade, Revestimentos cerâmicos portugueses - meados do século XIV à primeira metade do século XVI, Lisbonne, Edições Colibri / Universidade Nova de Lisboa, 2007 ; Luís Filipe Vieira Ferreira, David Soares Conceição, Diana Pereira Ferreira, Luís Filipe Santos, Tânia Manuel Casimiro et Isabel Ferreira Machado, « Portuguese 16th Century Tiles from Santo António da Charneca's Kiln: a Spectroscopic Characterization of Pigments, Glazes and Pastes ", Raman Spectroscopy, vol. 45, n 9, 2014, p. 838-847 ; Luís Filipe Vieira Ferreira, António Gonzalez et al., "Spectroscopy of 16th Century Portuguese Tinglazed Earthenware Produced in the Region of Lisbon », Ceramics International, vol. 41, n 10, 2015, p. 13433 13446 ; Susana Xavier Coentro, «An Iberian Heritage: Hispano-Moresque Architectural Tiles in Portuguese and Spanish Collections ", thèse de doctorat, Faculdade de Ciências e Tecnologia, Universidade Nova de Lisboa, 2017 [URL : run.unl.pt/handle/10362/24220].

10. Reynaldo dos Santos, O azulejo em Portugal, Lisbonne, Editorial Sul, 1957.

11. La Sé Velha de Coimbra a été bâtie dans la première moitié du XII ${ }^{e}$ siècle sur un édifice datable du IX $X^{e}$ siècle. Les éléments d'inspiration arabe y sont aujourd'hui peu nombreux, à l'exception de quelques motifs décoratifs du portail et d'une partie du revêtement original composé d'azulejos hispano-arabes de la fin du XVe siècle et du début du $X V l^{e}$, qui recouvraient les murs et les piliers des nefs.

12. Probablement construit sur la résidence des anciens wallis musulmans, datable du VIII' siècle, c'est le seul palais médiéval encore intact au Portugal. 
13. João Miguel dos Santos Simões, Azulejaria portuguesa no século XVIII (1979), Lisbonne, fondation Calouste Gulbenkian, 2010 ; José Meco, O azulejo em Portugal, Lisbonne, Publicações Alfa, 1989, p. 218.

14. Citons l'exposition dirigée par João Pedro Monteiro, "Um gosto português. O uso do azulejo no século XVII " (2012) et son catalogue, Um gosto português: o uso do azulejo no século XVII, cat. exp. (Lisbonne, Museu nacional do azulejo, 2012), Lisbonne, Athena, 2012.

15. João Miguel dos Santos Simões, Azulejaria em Portugal no século XVII (1971), Lisbonne, fondation Calouste Gulbenkian, 1997, 2 tomes ; dos Santos Simões, (1979) 2010, cité n. 13 ; Azulejaria em Portugal nos séculos XV e XVI, Lisbonne, fondation Calouste Gulbenkian, 1990.

16. Monteiro, 2012, cité n. 14 ; Maria Antónia Pinto de Matos et Alexandre Nobre Pais (dir.), Da Flandres. Os azulejos encomendados por D. Teodósio I, $5^{\circ}$ Duque de Bragança (c. 1510-1563) / From Flanders. The Azulejos Commissioned by D. Teodósio I, 5th Duke of Braganza (c. 1510-1563), Lisbonne, Direcção geral do património cultural / Museu nacional do azulejo, 2012.

\section{Monteiro, 2012, cité n. 14}

18. Sistema de referência e indexação de azulejo, recherche par motif [URL : redeazulejo.letras.ulisboa.pt/pesquisa-az/padrao_pesquisa.aspx] ; Rosário Salema de Carvalho, Rafaela Xavier, Inês Leitão, "Digital Techniques for the Study of Portuguese Azulejos (Glazed Tiles). Between Alice's White Rabbit and the Mad Tea Party", Kathryn Brown (dir.), The Routledge Companion to Digital Humanities and Art History, New York, Routledge, 2020, p. 408-419 [DOI : 10.4324/9780429505188].

19. Diana Gonçalves dos Santos, «Azulejaria de fabrico coimbrão (1699-1801). Artífices e artistas. Cronologia. Iconografia ", thèse de doctorat, Faculdade de Letras da Universidade do Porto, 2014.

20. Tiago Borges Lourenço, " $\mathrm{O}$ regresso à figuração no azulejo português da primeira metade do século XX (1890-1940) ", dans Maria Antónia Pinto de Matos et João Pedro Monteiro (dir.), Jorge Colaço e a azulejaria figurativa do seu tempo, Lisbonne, Museu nacional do azulejo, 2019, p. 24-36.

21. Ana Margarida Domingues Portela, "A ornamentação cerâmica na arquitectura do Romantismo em Portugal », thèse de doctorat, Porto, Faculdade de Letras da Universidade do Porto, 2009.

22. José Francisco Ferreira Queiroz, Os catálogos da fábrica das devesas, Lisbonne, Chiado Editora, 2016.

23. Michael Yonan, "Toward a Fusion of Art History and Material Culture Studies », West $86^{\text {th }}$ : A Journal of Decorative Arts, Design History, and Material Culture, vol. 18, n 2, Fall-Winter 2011, p. 232-248 [DOI : $10.1086 / 662520]$.

24. Rosário Salema de Carvalho, «A pintura do azulejo em Portugal (1675-1725): autorias e biografias - um novo paradigma ", thèse de doctorat, Universidade de Lisboa, 2012 [URL : repositorio.ul.pt/handle/10451/6527] ; Idem et Libório Manuel Silva, Azulejo em/in Braga - O largo tempo do Barroco / The Baroque Period, Vila Nova de Famalicão, Centro Atlântico, 2016 ; Idem, « To Be
Part of... Architecture, Decoration or Iconography. Documenting Azulejo as Integrated Heritage ", ISPRS Annals of Photogrammetry, Remote Sensing and Spatial Information Sciences, no IV-2/W6, 2019, p. 39-46 [DOI : 10.5194/isprs-annals-iv-2-w6-39-2019] ; Idem, « Les cadres des azulejos baroques portugais ", dans Nicolas Cordon, Édouard Degans, et al. (dir.), Jeux et enjeux du cadre dans les systèmes décoratifs de la première modernité, Rennes, Presses universitaires de Rennes, 2019, p. 59-72 ; Idem, « The Iconographic Role of Azulejo Frames ", Word \& Image, vol. 36, n 2, 2020, p. 135-165 [DOI : 10.1080/02666286.2019.1639347] ; Alexandre Nobre Pais, «Fabricado no Reino lusitano, o que antes nos vendeu tão caro a China. A produção de faiança em Lisboa, entre os reinados de Filipe II e D. João V ", thèse de doctorat, Universidade Católica Portuguesa, 2012 [URL : hdl.handle.net/10400.14/10123] ; Idem, "Clarividência. Os azulejos flamengos encomendados por D. Teodósio ", dans Jessica Hallett et Nuno Senos (dir.), De todas as partes do Mundo. O património do $5^{\circ}$ Duque de Bragança D. Teodósio I, Lisbonne, Tinta da China, vol. 1 - Estudos, p. 145-152 ; Idem et al., "The Beginning of the Production of Majolica Azulejos in Portugal - João and Filipe de Góis in 16th Century Lisbon ", Studies in Heritage Glazed Ceramics, $\mathrm{n}^{\circ} 1$, p. 1-23, [URL : glazedceramics.Inec.pt/SHGC_n01_.pdf].

25. « [Q]uem orquestrava todo este processo de forma cirúrgica ao ponto de oficinas lisboetas produzirem painéis imensos que eram aplicados a centenas ou milhares de quilómetros de distância sem a menor falha, como se, naturalmente, aí tivessem sido pintados? » João Miguel Simões, « Aspectos sociais dos artífices da arte do azulejo na cidade de Lisboa ", dans Monteiro, 2012, cité n. 14, p. 63.

26. Correia, 2018, cité n. 9.

27. Santos Simões, (1979) 2010, cité n. 13.

28. Sistema de referência e indexação de azulejo, recherche par localisation [URL : http://redeazulejo.letras. ulisboa.pt/pesquisa-az/imovel_pesquisa.aspx].

29. De Celso Mangucci, citons la thèse de doctorat en histoire de l'art, "História do azulejo, iconografia e retórica », Universidade de Évora, 2020 ; " A manufactura e a pintura de azulejos em Portugal da produção das primeiras faianças à grande indústria oitocentista », dans Rafael Salinas Calado (dir.), O revestimento cerâmico na arquitectura em Portugal, Lisbonne, Estar, 1998, p. 17-64; « Olarias de Louça e azulejo da Freguesia de Santos-o-Velho - dos meados do século XVI aos meados do século XVIII ", Al-Madan - Arqueologia, Património e História Local, II série, n 5, octobre 1996, p. 155-168. Il a aussi co-écrit, avec Rosário Salema de Carvalho, "Quem faz o quê: a produção de azulejos na época moderna (séculos XVI a XVIII) / Who does What: The Azulejo Production during the Modern Age (16th to 18th Centuries) ", ARTISON edição especial / Special Issue, $\mathrm{n}^{\circ}$ 6, 2018, p. 8-24.

30. Rosário Salema de Carvalho, "O Regimento do ofício de ladrilhadores da cidade de Lisboa », Revista de artes decorativas, $\mathrm{n}^{\circ}$ 5, 2011, p. 79-105.

31. Gonçalves dos Santos, 2014, cité n. 19.

32. Le LNEC fait paraître chaque année une revue qui publie une partie des résultats obtenus dans le cadre 
de ses recherches: Studies in Heritage Glazed Ceramics. On the Origin of Majólica Azulejos Production in Portugal, n 1 [URL : glazedceramics.Inec.pt/SHGC_ n01_.pdf] et Studies in Heritage Glazed Ceramics. On the Origin of Majólica Azulejos Production in Portugal, $\mathrm{n}^{\circ} 2$ [URL : glazedceramics.Inec.pt/SHGC_n02_.pdf].

33. Groupe de recherches de I'ARTIS - Instituto de História da Arte, Faculdade de Letras, Universidade de Lisboa.

34. Projecto de salvaguarda e valorização do património azulejar português [URL : sosazulejo.com/].

35. Rede de investigação em azulejo [URL : redeazulejo.letras.ulisboa.pt/].

36. Rosário Salema de Carvalho et Libório Manuel Silva (dir.), Azulejos - Maravilhas de Portugal / Wonders of Portugal, Vila Nova de Famalicão, Centro Atlântico, 2017.

37. Azulejos. Chefs-d'œuvre du Musée national de l'azulejo, Paris, Chandeigne, 2009 ; Les Fables de La Fontaine du monastère de Saint-Vincent à Lisbonne, Paris, Chandeigne, 2008 ; Pascal Quignard, La frontière : azulejos du palais Fronteira (1992), Paris, Chandeigne, 2003.

38. Mentionnons la création récente de I'AIAI Associação para a interpretação do azulejo industrial, dont le but est de traiter, de collecter et d'organiser des exemplaires d'azulejos industriel produits au $\mathrm{XX}^{\mathrm{e}}$ siècle. 